\title{
An Interactive Web-based Decision Support System for Mass Dispensing, Emergency Preparedness, and Biosurveillance ${ }^{*}$
}

\author{
Eva K. Lee ${ }^{\dagger}$ \\ Georgia Institute of Technology \\ Atlanta, Georgia 30332 \\ Chien-Hung Chen \\ Georgia Institute of Technology \\ Atlanta, Georgia 30332
}

\author{
Ferdinand H. Pietz \\ Centers for Disease Control and Prevention \\ Atlanta, Georgia 30333 \\ Yifan Liu \\ Georgia Institute of Technology \\ Atlanta, Georgia 30332
}

\begin{abstract}
In this study, we present an interactive web-based real-time decision support suite, $\operatorname{RealOpt\odot }{ }^{\odot}$. The system integrates visualization, information and cognitive analytics, and dynamic large-scale computational modeling and optimization tools that allow public health emergency preparedness coordinators to determine optimal response facilities and locations, resource needs and supply-routes, and population flow in real time. With an eye towards flexibility and future system expansion, RealOpt is designed in modular format allowing direct linkage to multiple functional modules. Currently, the system has twelve modules covering emergency response preparedness and operations for biological, chemical, radiological/nuclear incidents, biosurveillance, epidemiology, and decontamination models, operations logistics and networks, a real-time crowd sourcing data feed, and evacuation planning. RealOpt has been used for biodefense and H1N1 regional planning and operations, regional flood and hurricane responses, 2010 Haiti earthquake disaster relief, 2011 Japan Fukushima disaster, 2014-2015 Ebola containment assistance and after-event public health preparedness training in West Africa, and current Zika virus containment analysis. The fast solution engines enable real-time use for rapid decision and scenario analysis, since it requires only one CPU minute to determine an optimal network of facilities and resource needs to serve a population of over 10 million.
\end{abstract}

\section{CCS CONCEPTS}

-Information systems $\rightarrow$ Decision support systems; •Computing methodologies $\rightarrow$ Model development and analysis; $\bullet$ Applied computing $\rightarrow$ Health care information systems;

\footnotetext{
*The findings and conclusions in this report are those of the authors and do not necessarily represent the official position of the CDC

${ }^{\dagger}$ Corresponding author. eva.lee@gatech.edu
}

\footnotetext{
Permission to make digital or hard copies of part or all of this work for personal or classroom use is granted without fee provided that copies are not made or distributed for profit or commercial advantage and that copies bear this notice and the full citation on the first page. Copyrights for third-party components of this work must be honored. For all other uses, contact the owner/author(s).

DH '17, London, United Kingdom

(c) 2017 Copyright held by the owner/author(s). 978-1-4503-5249-9/17/07. DOI: http://dx.doi.org/10.1145/3079452.3079473
}

\section{KEYWORDS}

Emergency response; real-time decision support; mass dispensing; simulation-optimization; facility location optimization; disease modeling; network analysis; agent-based modeling.

\section{ACM Reference format:}

Eva K. Lee, Ferdinand H. Pietz, Chien-Hung Chen, and Yifan Liu. 2017. An Interactive Web-based Decision Support System for Mass Dispensing, Emergency Preparedness, and Biosurveillance. In Proceedings of DH '17, London, United Kingdom, fuly 02-05, 2017, 10 pages.

DOI: http://dx.doi.org/10.1145/3079452.3079473

\section{INTRODUCTION}

Planning for a catastrophe involving a disease outbreak or mass casualties $[3,7,27]$ is an ongoing challenge for first responders and emergency managers. They must make critical decisions on treatment distribution points and staffing levels, and gauge the potential impact on affected populations in a compressed window of time when seconds could mean life or death. Although extensive efforts have been made to plan for a worst-case scenario on the local, regional and national scale, the U.S. Government Accountability Office (GAO) found gaps still exist [10]. While many states have made progress in planning for mass casualty events, many have noted concerns related to maintaining adequate staffing levels and accessing other resources necessary to effectively respond.

After outbreaks of potential public health emergencies, immediate and aggressive responses must be carried out. This includes medical countermeasures dispensing $[14,30]$ to mitigate possible deaths and to control epidemic. Besides dispensing medication, "service constructs" for food and water distribution, temporary shelters, medical care, screening and registry, and decontamination may also be required. Without loss of generality, we call such sites point-of-dispensing sites (PODs). These are the places where affected individuals come to receive services.

While dispensing medical countermeasures and providing medical services require specific healthcare personnel, distribution of food and water and other personal needs can be carried out by other emergency workers and volunteers. Nonetheless, both types of services share key elements: resources are scare, time is precious, risk is uncertain and evolving, there is a large affected population to serve, and the on-the-ground conditions can be exceedingly stressful, for the impacted population and for the emergency workers. To maximize the throughput that can be served under limited resources of labor, time, and potential damaged infrastructure, PODs 
must be established such that they are flexible, scalable, sustainable, and agile for operations continuity and fluidity. Policy and decision makers must determine where to establish a network of sites to achieve the maximum overall benefit. Further, POD layout must be designed to facilitate the best operations workflow and throughput while minimizing resource requirements.

CDC's Cities Readiness Initiative (CRI) is designed to enhance preparedness in the nation's largest population centers to effectively respond to large-scale public health emergencies needing life-saving medications and medical supplies. State and large metropolitan public health departments develop, test, and maintain plans to quickly receive medical countermeasures from CDC's Strategic National Stockpile and distribute them to local communities [8]. RealOpt $\odot$ is designed and used by many such departments to assist in these efforts.

For a potential anthrax response, the goal of dispensing countermeasures to the affected population within 48 hours of dispensing decision presents real challenges. A commercial system that offers a discrete event simulation tool for scenario analysis, but takes over 10 hours to generate a feasible solution per scenario, is not suitable for real-time planning [25]. In contrast, scenario analysis and resource optimization in RealOpt is typically completed under one minute, so is well-suited for real-time planning.

RealOpt is an interactive online software enterprise for largescale regional medical dispensing and emergency preparedness and responses. It features interactive visualization tools to assist with spatial understanding of important landmarks in the region, assess the population densities and demographic makeup of the region, and identify potential facility locations. It also features backend mathematical models for large-scale facility location, resource allocation optimization, and dynamic disease propagation problems. The system is equipped with novel rapid solution engines for strategic and operational planning, real-time dynamic optimization, and epidemiological analysis.

In the following sections, we first describe the system architecture and design of RealOpt. Next, the system modules are presented, beginning with functionalities that relate to the user experience, and moving on to functionalities that pertain to managing geographical boundaries; facility location and population assignment; ZIP code and population composition; multi-modality dispensing; POD layout design and resource allocation; disease propagation analysis and biosurveillance; and regional information sharing, reverse reporting, tracking and monitoring, and resupply. Application of the system to planning for mass dispensing for anthrax response, and to planning and responding to actual disasters will be briefly presented. The paper concludes with a discussion of future work and challenges.

\section{SYSTEM ARCHITECTURE AND DESIGN}

With flexibility and expansion in mind, RealOpt is designed in modular form (Figure 1) and allows direct linkage to functional modules. Currently, it is composed of twelve primary modules for integrating system interactivity, client-server architecture, optimization, and policy/decision support. The foundation web-component in the systems design is the RealOpt-Regional@ module.

RealOpt-Regional@ is implemented via the client-server architecture. For clients, the Google Maps API [9] along with a webpage are incorporated to provide the primary I/O functionalities. On the server, a rapid hybrid large-scale algorithm that couples features of a combinatorial genetic algorithm and an adaptive greedy approach is implemented as a solution engine for optimization of the network of facility sites and population flow and assignment [22]. The intensive optimization computation is performed on the server, and requires no extra computing effort from the client. The user's system is used primarily for webpage display and object rendering on the map.

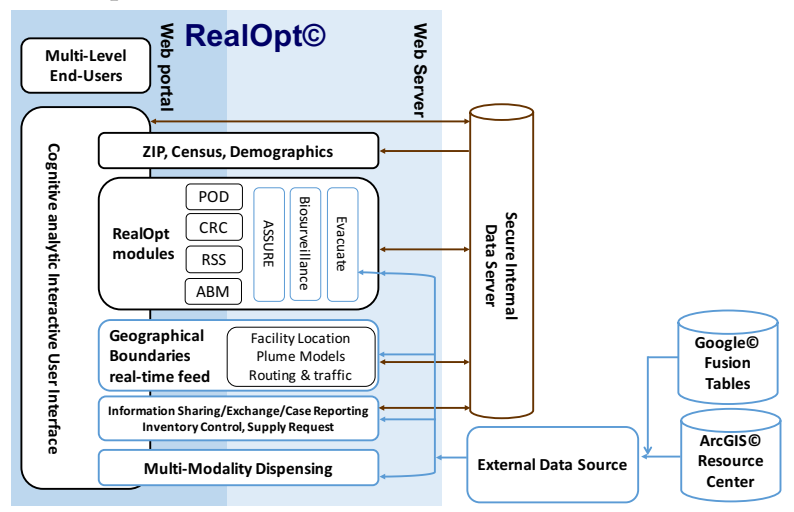

Figure 1: Some of the system modules inside the RealOpt $\odot$ Suite.

Asynchronous JavaScript and XML (AJAX) with basic access authentication is used for sending and receiving requests asynchronously. In RealOpt-Regional, a large-scale facility location problem may take seconds or a minute to initiate and solve. AJAX enables sending the optimization request from the client to the server wherein users do not have to wait for response from the server during optimization. Users at this point are free to explore other features in RealOpt-Regional. Further, even if the client becomes disconnected from the server, the user can still retrieve the optimization results. Potential internet disruption is unavoidable; hence this feature is very appealing and important. While the connection can be manually or automatically re-built, the running progress (and solutions) will not be accessible unless another request is sent from client web browser to the server. AJAX enables users to obtain updated running progress or optimization results once the program runs to completion.

Java Native Interface (JNI) is employed to link multiple modules on the server to complete the backend computation and interface. The system architecture is designed so that only the junction module needs modification when updating the solution engine. A schematic design of the RealOpt-Regional system architecture is depicted in Figure 2.

\section{SYSTEM MODULES \& FUNCTIONALITIES}

We briefly describe some of the RealOpt $\odot$ system modules and functionalities below.

\subsection{Interactive User Experience}

We incorporate the graphical interactivity enabled by Google Maps JavaScript API as part of the I/O functionalities. With up to 100,000 map loads per 24 hours, Google Maps JavaScript API provides an efficient and stable mapping environment for users. This module 


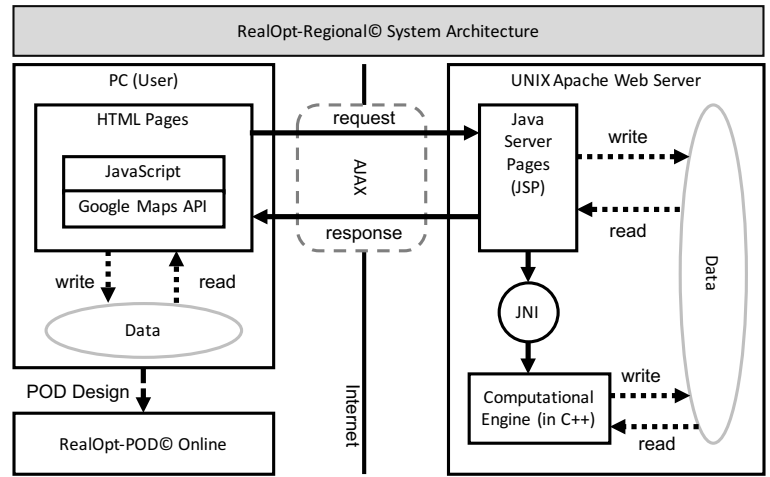

Figure 2: A schematic design of the RealOpt-Regional@ system architecture.

equips users with spatial understanding of important landmarks in the planning and surrounding regions. Also, without the need for prerequisite knowledge in mathematical programming, users are empowered to build, optimize, and evaluate service networks in a graphical visualization environment. They can interact with the map by selecting jurisdictions, adding/removing potential PODs (as markers), defining dispensing constraints (e.g., capacity constraints in each facility, and travel distance/time constraints), and highlighting population served, household characteristics and various parameters (e.g., average number of members per family; ethnicity/language represented in the population). In the backend, the associated mathematical model will be built automatically and sent to the server for optimization.

Other capabilities are implemented to allow users to manage their files on the secured server directly from their web browser (e.g. adding, saving, uploading, and removing). This creates a user experience like that of using a PC-application even though it is in the web environment. The interactivity is designed to be compatible with mobile tablet devices. Figure 3 shows the interface of RealOptRegional for public health emergency preparedness coordinators and planners. On the left is a Google map, and on the right are six function panels: "Jurisdiction", "POD (Marker)", "Optimization" (Facility Location), "DrawPolygon”, "Multimodes", and "Twitter". Help files and step-by-step practice scenarios are provided to guide users in building their own planning cases and to familiarize them with usage of the system.

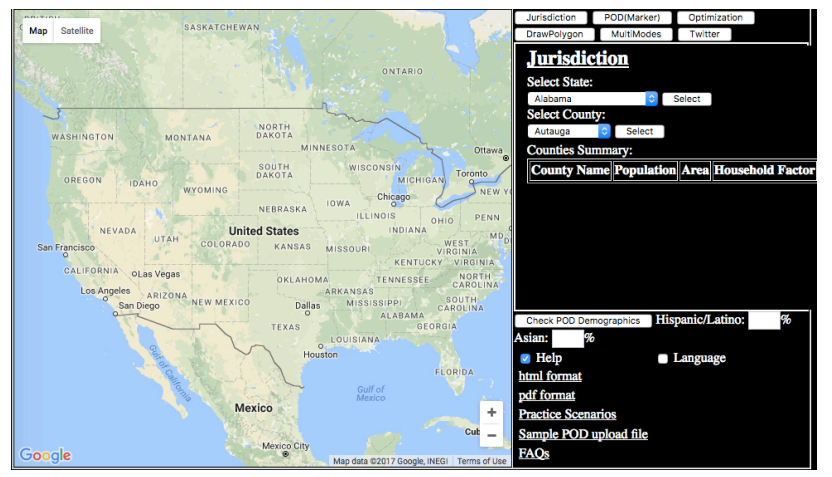

Figure 3: Interactive user interface combining visualization, graph drawing, modeling analytics, and real-time data pull and computational solution engines.

\subsection{Geographical Boundaries}

Through ArcGIS [5], RealOpt-Regional can display the geographical county boundaries of the United States. Emergency response planners can select and define their planning area spatially. Demographics such as population and density of each county are also provided to complete the necessary input when planners are building and optimizing the dispensing / service networks.

City boundaries can be defined by free drawing polygon tools "DrawPolygon" on the map. This enables RealOpt-Regional to work compatibly with the Cities Readiness Initiative to enhance preparedness of our nationfis largest cities and metropolitan statistical areas. The flexibility of users manually drawing planning areas also ensures that the heterogeneity of population densities between cities and counties can be considered in building the dispensing networks. Further, such a free drawing feature is critical for international sites (e.g., the disaster response effort for the 2010 Haiti earthquake) where populations are on the move, and affected regions are dynamic. The drawing process, which includes tools for adding/deleting a vertex, and removing and modifying the polygon shape, simply amount to a series of clicking and dragging operations on the map. Users can save their drawings for future use. Figure 4 illustrates an example use of "DrawPolygon" in outlying the boundary of the city of Chicago.

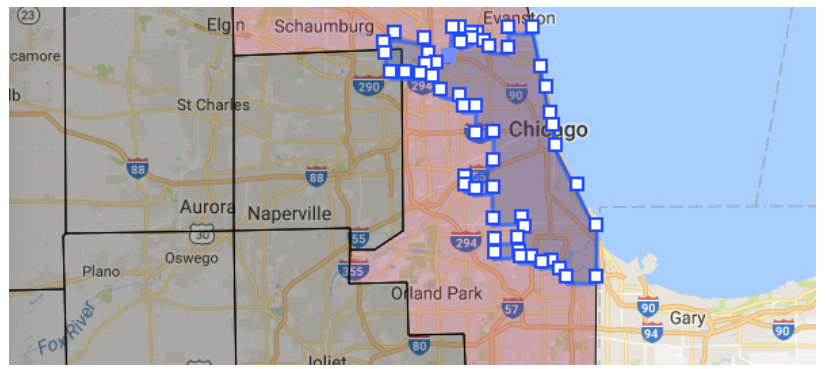

Figure 4: Simple click-and-drag to obtain a user-defined boundary of the city of Chicago.

\subsection{Network of Service, Locations and Population Flow and Assignment $[16,22,23]$}

Once a region is selected/drawn, users can input potential PODs by loading a file with addresses, input the addresses interactively, or simply drag-and-drop the POD markers onto the region. Physical constraints on the facility must be modeled, e.g., capacity of a facility cannot be violated (e.g., parking capacity, fire codes, and building regulations limit the total number of individuals that can be present simultaneously inside a facility). Users can specify this globally for all PODs, or more commonly, they can specify the value for each POD. Travel distance, household factors, and time for completing services at the facilities can also be input.

Various objectives are incorporated within the RealOpt-Regional computational engines. In the event of catastrophic incidents, it is critical that PODs are strategically located to allow easy access by the affected public. Hence, minimizing transportation time and distance is one critical objective. Further, the setup and operating costs of PODs cannot be neglected. In our model, setup costs and travel time form the composite objective criteria. 
A POD must be accessible by service workers. It should include a good communication infrastructure, and must be readily protected by law-enforcement personnel. The facility must be capable of handling a large influx of people with good access routes for supply and re-supply. The spatial maps allow the users to evaluate the surrounding POD areas to ensure that candidate POD selections are deemed appropriate.

The planning area may include a set of counties and/or a composition of a free drawing region. By default, it is discretized at 40 -by-40 resolution. The population within the planning area is assumed to be distributed evenly, or it can be strategically specified.

Once the set of potential PODs are selected with desired parameters, RealOpt-Regional translates this input into a facility location problem on the backend. Specifically, given a selected jurisdiction and a regional population, under the capacity and travel distance constraints, setup cost and travel distance/time as objectives, and the required completion time for protecting the chosen regional population, we must determine a) an optimal set of PODs and their respective locations needed for cost-effective operations; and b) the population assignment to this optimal set of PODs.

The computational engine consists of a two-stage facility location integer program. The first stage optimizes the number of facilities opened. This is formulated as a capacitated POD-location problem COVER-CAP to ensure that at least two PODs are opened. If a catastrophic event at one site necessitates shutting down a POD, the remaining location can continue to carry out the emergency dispensing. The model ensures that each household will travel at most the maximum allowed travel distance and time, every household is served, and that the capacity of the facility is not violated. COVERCAP returns the minimum total number of PODs needed. Next, the MINAVG-CAP problem is formulated to minimize the travel distance and time over all households while keeping the number of PODs fixed to the optimal value that the COVER-CAP problem determined. Lee et al. [16] and Lee et al. [23] describe variations of the full IP formulations.

For a jurisdiction of about 20,000 households, current competitive commercial optimizers fail to solve any of the instances after running for several months of CPU time. The difficulty is partly because facility location remains an NP-hard problem and partly due to the diverse requirements of the facilities and the very mixed population densities. Using recent computational advances for solving intractable facility-like instances [24], we were able to solve instances ranging from 200,000 to 10 million variables within 40,000 CPU seconds. To facilitate strategic and operational planning and real-time dynamic decision analysis, we design a novel rapid solution engine that couples a combinatorial genetic algorithm with an adaptive greedy search [22]. The optimization heuristic schema is shown in Figure 5 . The engine is implemented in $\mathrm{C}++$. Algorithmically, users can wait until the optimization is completed (usually takes only seconds), or they can interrupt the solution process manually to obtain intermediate non-optimal solutions.

\subsection{ZIP Code and Population Composition}

By rearranging and analyzing ZIP code demographics and area boundaries [28, 31], RealOpt-Regional maintains a set of up-todate census data bank that contains 32,036 postal-code areas in

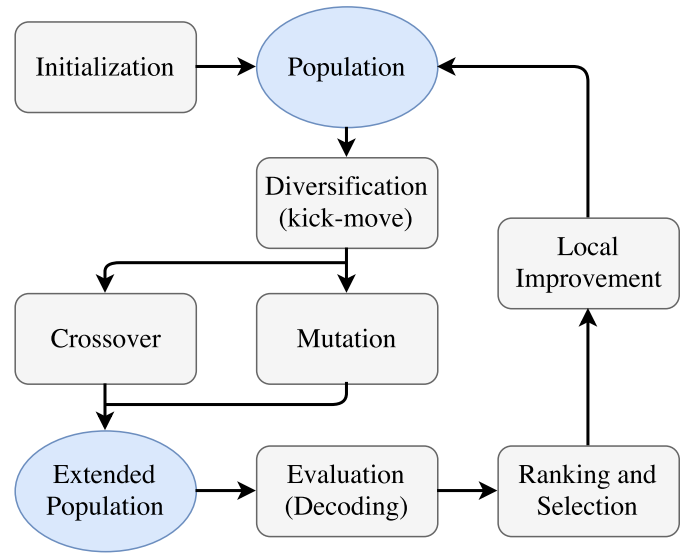

Figure 5: Algorithmic flow of our facility location heuristic.

the United States. The system provides ZIP code based information including regional demographics, social indicators, economic indicators, and boundary of ZIP code areas.

After determining a set of optimal POD locations and population assignment, users can query POD coverage based on demographics by overlaying the POD service area with all adjacent ZIP code areas, and aggregating ZIP code based data by considering size of overlapping, and population density of each overlapped ZIP code area. POD coverage based demographics are dynamic and dependent on user-defined parameters in optimizing the locations and the total number of facilities. Using this information, emergency planners can identify appropriate personnel for each specific POD and/or mode of dispensing (e.g. pediatric assistants, translators, transportation needs or mobile PODs for socially-disadvantaged individuals). Figure 6 and Figure 7 illustrate the population composition by overlapping POD coverage with all adjacent ZIP code areas. We can identify demographics of the majority population served by the POD. Different icons can be used to distinguish POD types accordingly.

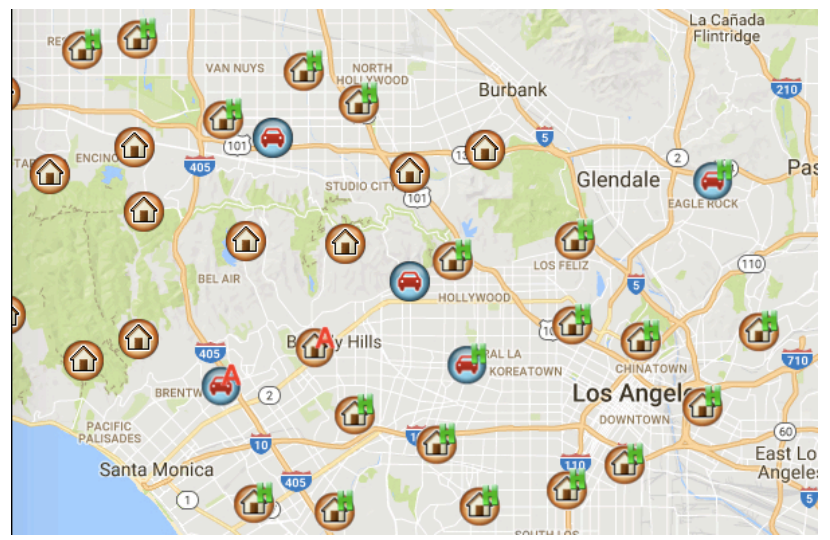

Figure 6: A network of PODs serving the City of Los Angeles returned by RealOpt-Regional. Each is marked according to the demographics of the majority population it serves, and mode of operations (drive-through versus walkthrough). "H" stands for "Hispanic/Latino", and "A" stands for "Asian". 


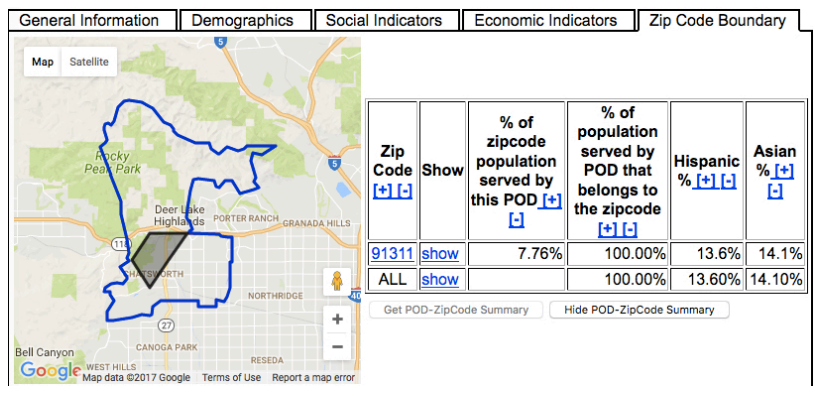

Figure 7: POD demographics for Shadow Oak Dr, Los Angeles, CA 91311.

\subsection{Multi-Modality Dispensing \& Public-Private Partnership}

The goal of mass dispensing is to protect the worried well (general population) efficiently and effectively under time constraints. In an anthrax attack, it is important that the affected population receive antibiotic prophylaxis within 48 hours of the determination that an attack has occurred, since the mortality rate for persons demonstrating symptoms of inhalation anthrax is extremely high [15]. Multiple dispensing modalities often must be employed to serve the entire regional population rapidly $[16,23]$.

Open PODs (i.e., open to the public) can be drive-through or facility-based walk-through. Special needs populations may need closed PODs on site. For example, nursing homes, assisted living facilities, homeless shelters, hospitals, and prisons house many residents for whom it would be inconvenient or inadvisable to travel to a public dispensing facility.

Further, self-organized closed PODs should be promoted. Corporate offices with many employees could protect their own. Once these sites receive prophylactic supplies, they could set up a closed POD with their own healthcare staff and volunteers, or with public health staff supplemented by the state. Several factors suggest that such closed PODs will have fewer security concerns and will be easier to manage than public PODs. These factors include familiarity with the environment and people (e.g., fellow residents/employees), existing security measures including established checkpoints and previously authenticated identification badges with photo and/or biometric markers, and less stress than having to commute to a public POD.

Airports and hotels where many non-resident travelers can be found are also candidates for setting up PODs. This is particularly important with infectious disease outbreak to ensure that travelers are protected with medical countermeasures before they leave for elsewhere. Universities can use their own health facilities (and if necessary, additional mobile on-campus PODs provided by the state) to provide prophylaxis to on-campus students, staff and faculty. Clearly, if large employers and medical facilities provide prophylaxis to their own employees, families, and patients, it will eliminate a high percentage of the population (may be as high as $40 \%$ in some large cities) from visiting public PODs, thus reducing the burden on these facilities.

POD markers in RealOpt-Regional can be marked as closed or open, drive-through or walk-through, and with specific demographics needs. Currently the system maintains a data bank of 2,000 homeless shelters [12], 4,200 universities and colleges [29], 5,128 airports [2], 12,881 high schools [4, 11], 73,079 assisted living facilities [6], and 24,192 hotels [13]. Public health and emergency response planners are equipped with this information and can make better decisions in constructing more cost-effective and efficient mass dispensing networks. Users can also search specific business or facility types to explore options of dispensing sites.

\subsection{POD Layout Design and Resource Allocation}

RealOpt-POD॰ $[20,21]$ was the first RealOpt module. It was designed and implemented in 2003, and has been advanced through the years. It currently has over 10,000 public health site users. Its users also include fire departments, police departments, and hospital networks. Written entirely in Java, it is a stand-alone computerized decision support system for facility layout, resource allocation, and intra-facility disease propagation analysis. The realtime solution capability is supported by automatic graph drawing tools, and our in-house large-scale simulator and optimizers. Since 2009, a branch version has been developed within the RealOpt web-based client-server architecture. It is also interfaced with RealOpt-Regional. While the automatic graph-drawing tool and user interface are built into applets and embedded in the webpage, just as RealOpt-Regional, the core computation for optimization and large-scale simulation are powered by the server. Figure 8 shows the web-user interface of RealOpt-POD.

The linkage of RealOpt-POD and RealOpt-Regional allows users to design POD layouts and perform resource allocation optimization on the spot once the network of facilities is determined. However, the standalone version RealOpt-POD is still in high demand since planners do not need internet access to use it.

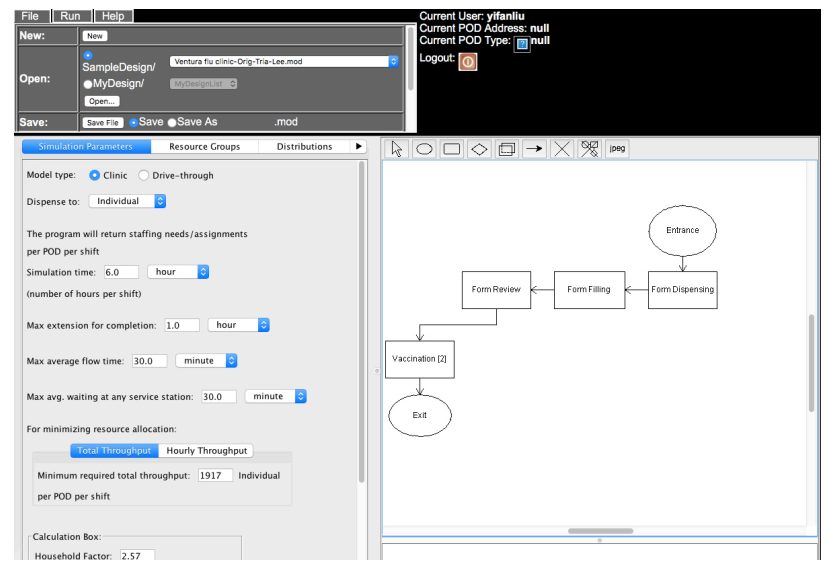

Figure 8: RealOpt-POD® Online for POD design, resource allocation and disease spread epidemiology analysis.

\subsection{Radiological Module}

RealOpt-CRC@ is designed in parallel to RealOpt-POD for population screening in response to radiological/nuclear incidents. There are major overlaps in these two systems wherein CRC incorporates the decontamination, bioassay and other processes that are critical for radiological screening and population registry and protection. It was used in response to the 2011 Fukushima Daiichi radiological disasters (See Section 4.2.2). 


\subsection{Biosurveillance}

Large-scale dispensing of medical countermeasures has proven to be an effective way to contain outbreaks of highly infectious diseases, such as smallpox, pneumonic plague, and pandemic flu $[14,30]$. Further, successful dispensing operations, such as optimizing POD operations through RealOpt-POD, can help reduce resource requirements, shorten patient waiting time and queues, and maximize throughput $[21,22]$. However, the large influx of individuals brought into these dispensing centers raises the potential risk of intra-facility cross-infections.

RealOpt supports surveillance of biological attacks or natural pandemics on three fronts.

a. Intra-infectivity tracking: In the planning phase of a dispensing event, POD planners and epidemiologists can employ RealOptPOD for estimating the dynamics of intra-POD infection under different scenarios of POD design and personnel allocation. The epidemiology module integrates agent-based simulation within the discrete event simulation platform, offering a powerful means to track an individual's health status within the dispensing operations. Figure 9 illustrates the disease spread and infectivity dynamics within POD via the novel 6-stages SEPAIR disease spread model [17].

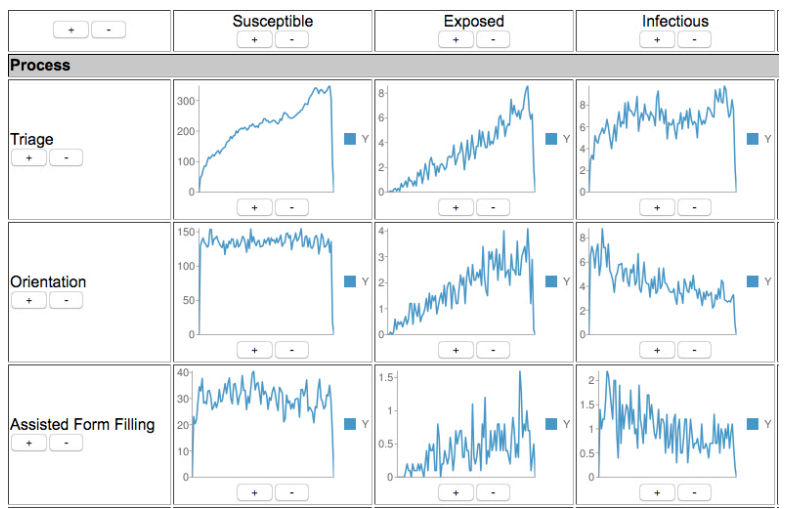

Figure 9: Tracing the dynamics of intra-facility infection (at each service station) within the RealOpt system.

b. Case incidents tracking: In addition to providing the epidemiological estimates during the planning stage, POD managers can report and register incidents via RealOpt in real time during the dispensing event. These numbers, along with the attached timestamps, can provide valuable information in monitoring and containing the disease spread. They are also beneficial for epidemiologists to collect data and refine model parameters in real time.

c. Incorporation of plume models within regional planning. RealOptRegional allows automatic import of plume models and biologicalinfected regional zones within the planning region. Users can also import plume radius and coverage via the free drawing panel. This information can be critical for population protection planning, allowing planners to select proper/safe candidate sites (for shelters, medical service, and/or dispensing) and estimate resources required for regional coverage.

\subsection{Regional Information Sharing, Reverse Reporting, Tracking and Monitoring, and Resupply}

Beyond strategic and operational planning, training and cost-effectiveness analysis, RealOpt also offers various information sharing/exchange and tracking capabilities. Specifically, it allows users to:

Input medical supply burn rates. The data will be registered with a timestamp. This allows regional commanders to oversee the dispensing process across the affected region, re-direct populations as necessary, and order re-supplies appropriately.

Perform real-time POD reconfiguration using current service performance information through the service distribution estimator in RealOpt-POD.

Report case incidents for disease monitoring and derivation of mitigation strategies.

Perform reverse information sharing by highlighting traffic and road conditions in surrounding areas to alert regional managers of necessary steps to re-route transportation or re-direct citizens.

Input plume information to alert populations of potential risks and guide them to stay outside the plume zone(s).

The information can be used by multiple stakeholders at different levels. Regional commanders are empowered with multi-level ground knowledge and performance measurements that will facilitate expediting operations, mobilizing resources, and containing threats and spread of disease.

\subsection{Multi-Level End-Users Access}

RealOpt can be used by both emergency preparedness personnel and the public to obtain service site information. Multiple levels of authorization are used to control proper access. In general, emergency preparedness personnel can:

- Determine a network of optimal service/dispensing locations and the optimal combination of dispensing modalities (e.g. combinations of drive-through, walk-through, mobile, open and closed PODs) for regional preparedness.

- Review demographics at each site and identify the appropriate personnel (e.g. translators, pediatric assistants) needed for effective dispensing.

- Determine in real-time a resupply schedule for medical countermeasures across the network through real-time feeds of product usage.

- Determine the closest facility and feasible traffic routes (incorporating inverse road conditions) to resupply, and to transport sick individuals from PODs to respective hospitals for treatment.

- Identify region-wide budget and labor needs to accommodate an emergency, and determine recruitment needs of healthcare workers and volunteers.

- Perform economic analysis on multi-modality variations.

Emergency preparedness personnel can be further categorized into three levels: "emergency planner", "RSS (receive-sort-staging) manager", and "regional commander". Emergency planners are provided with general functionalities mentioned above; RSS managers can model RSS sites on the map, and can provide supply when requested. Regional commanders can further manage inventory at each site, review inventory summary over all sites, review item 
information summary, and manage shipments whenever necessary. Individual POD managers can login to design POD layout and optimize POD performance via RealOpt-POD. They can register/report burn rates, case incidents, and request resupply. Epidemiologists work along POD and Regional commanders to analyze disease spread and design mitigation strategies.

The system will be open to the public only during an actual event to obtain driving directions to primary and backup PODs that are specifically assigned to them. They can also review relevant information related to the specific medical countermeasures dispensed, and to register for the medication.

\section{BIODEFENSE, PANDEMIC PREPAREDNESS PLANNING, RADIOLOGICAL AND LARGE-SCALE DISASTER RELIEF EFFORTS}

RealOpt has been used for planning for mass dispensing for anthrax response, actual vaccination events for seasonal flu, 2009/2010 H1N1 response, and hepatitis $\mathrm{B}$ vaccination. The system has also been tailored for the 2010 Haiti earthquake emergency response, the 2011 Japan Tohoku-Fukushima radiological disaster efforts, the 2014 Ebola response and training operations, and the current Zika virus strategic containment analysis.

\subsection{Biodefense Mass Dispensing Regional Planning}

To illustrate the strategic capability and importance of RealOpt, we briefly describe the biodefense planning and capability and resource requirement estimates for New York City. The city consists of five counties: Bronx County (Bronx), Kings County (Brooklyn), New York County (Manhattan), Queens County (Queens), and Richmond County (Staten Island). According to the U.S Census Bureau, the total population in New York City was estimated to be 8,550,405 in 2015. The land area was 303 square miles, and the number of persons per square mile is 27,603 . Among the entire population, $47.60 \%$ speak languages other than English at home.

For brevity, we illustrate only one analysis here where the maximum travel distance is set to 15 miles, and the physical capacity of each facility ranges from 1,000 to 2,000 per hour, reflecting the actual site specification. Two 12-hour shifts are operating at each location to ensure that the population is protected within 24 hours. Using census data, it is assumed that an average household has 2.57 people.

Figure 10 shows a POD network returned by RealOpt-Regional that covers $96.3 \%$ of the New York City population. A total of 101 PODs are needed, each runs two 12-hour shifts. Figure 11 illustrates the percentage of non-English speaking (Hispanic/Latino and Asian) population served by one of these POD locations. Using this information, emergency planners can contrast the type and number of interpreters needed to execute the necessary POD operations.

Next RealOpt-POD is used to design POD floorplan and predict the optimal resource allocation, based on the POD operations service distributions collected from local jurisdiction. Figure 12 shows a typical walk-through POD layout used in New York City. Figure 13 shows the number and types of workers needed to cover $96.3 \%$ of New York City population with or without splitting the PODs into Hispanic-operated versus non-Hispanic operated. The number

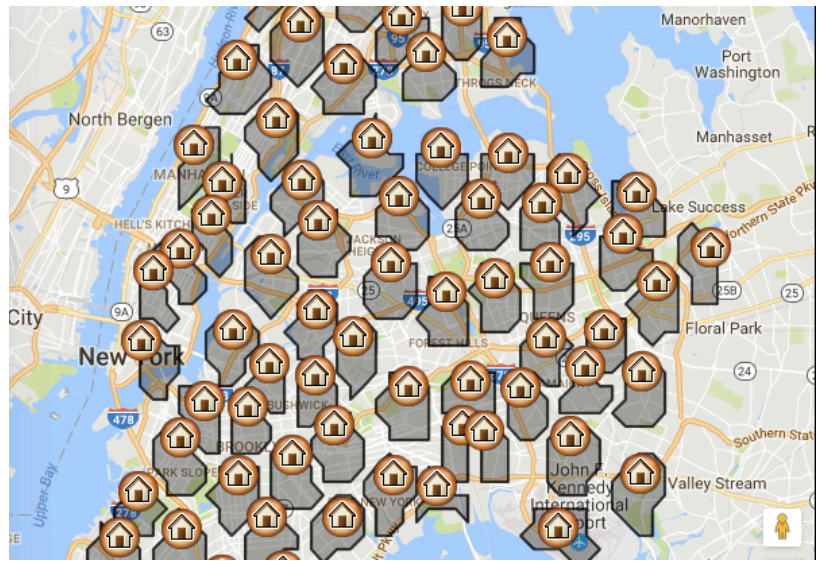

Figure 10: Optimal POD facility network for New York City.

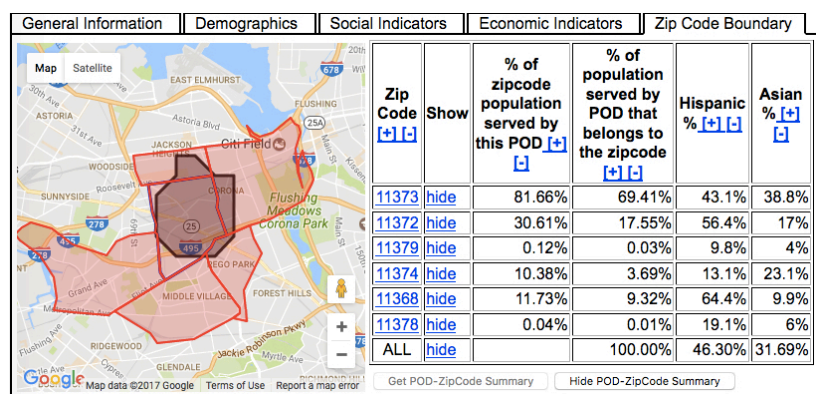

Figure 11: ZIP code-based demographical information.

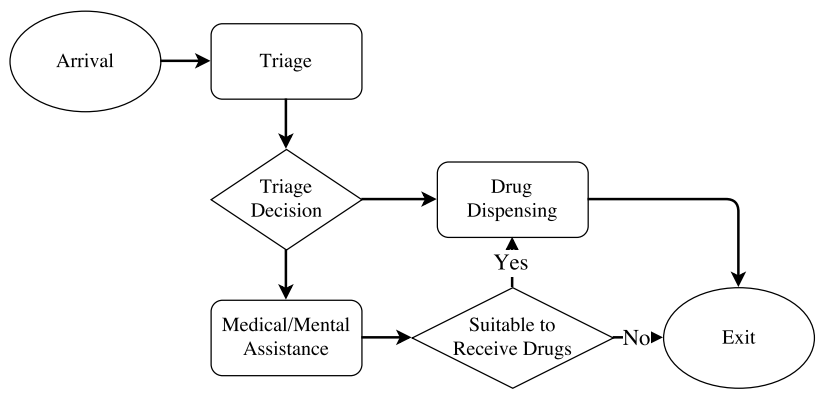

Figure 12: A layout for a walk-through POD.

of interpreters in the splitting case is significantly smaller than the non-splitting case. This indicates that the PODs in the splitting case are easier to set up (for just-in-time training and managing) for efficient operation during emergencies.

\subsection{Real-Life Disaster Response Effort}

Large-scale disaster and humanitarian relief efforts (e.g., in response to earthquakes, hurricanes, forest fires) where homes are destroyed, critical infrastructures are damaged, and tens of thousands or millions of people's lives are affected, require rapid establishment of "service constructs". These service constructs serve as camp shelters for the displaced population; as distribution nodes for receiving supplies for on-the-ground responders; as dispensing sites for handing out food and water to the affected population; and as hospital tents for medical care of the injured and sick. 


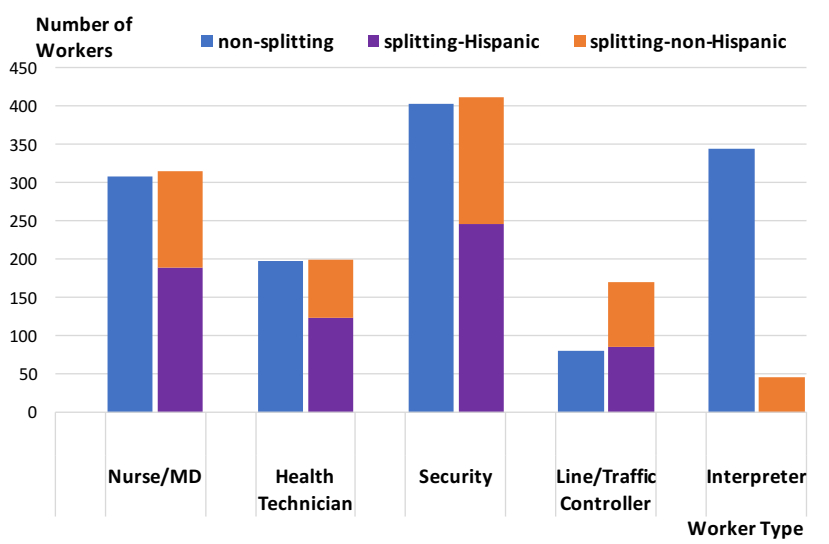

Figure 13: Total number and types of workers needed to cover $96.3 \%$ of New York City Population: contrasting staffing needs of splitting versus non-splitting POD operations.

4.2.1 RealOpt-Haiti@. In 2010, RealOpt was tailored to RealOptHaiti@ for part of Haiti's emergency response efforts. In this case, distribution nodes were established, with affected populations estimated by region based on on-the-ground dynamics and movement of the population. Figure 14 shows the supplies arriving at the airport and seaport, and the population density across the 11 in-land distribution nodes. The system allows distribution optimization and provides the responders the items and quantities of shipment to each distribution node. Figure 15 shows the distribution paths and supply items and quantities from the supply nodes (airport and seaport) to the in-land distribution nodes returned by RealOpt-Haiti. The system was also used in optimizing resource allocation and operations within each distribution node, temporary medical tents, and camps/shelters for the displaced population.

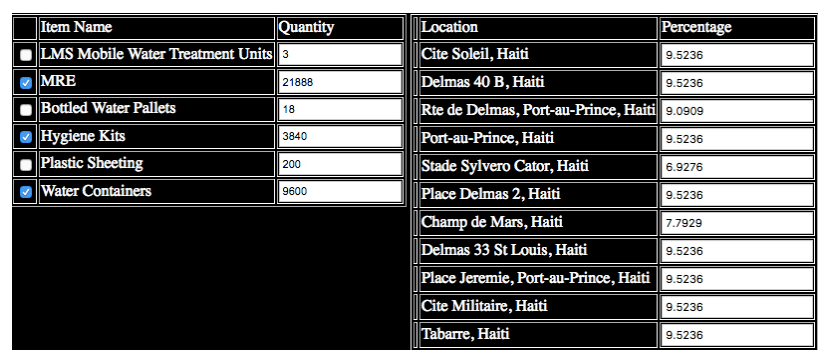

Figure 14: Supplies at airport and seaport and the estimated population density across the distribution nodes.

4.2.2 RealOpt-Regional and RealOpt-CRC for Fukushima Daiichi Nuclear Disaster. The Tohoku earthquake, tsunami, and subsequent radiological incidents present a glimpse of the devastating impacts of cascading failures and a catastrophic event in an advanced industrialized society. This is the first major hydrologic release of radiation isotopes and is the largest-ever release to the oceans. The cause was not a single event, but rather a cascading failure and the release persisted for a sustained period.

In Japan, while citizens have been educated about evacuation and response procedures for earthquakes and tsunamis, there is a serious knowledge gap regarding strategies and emergency guidelines

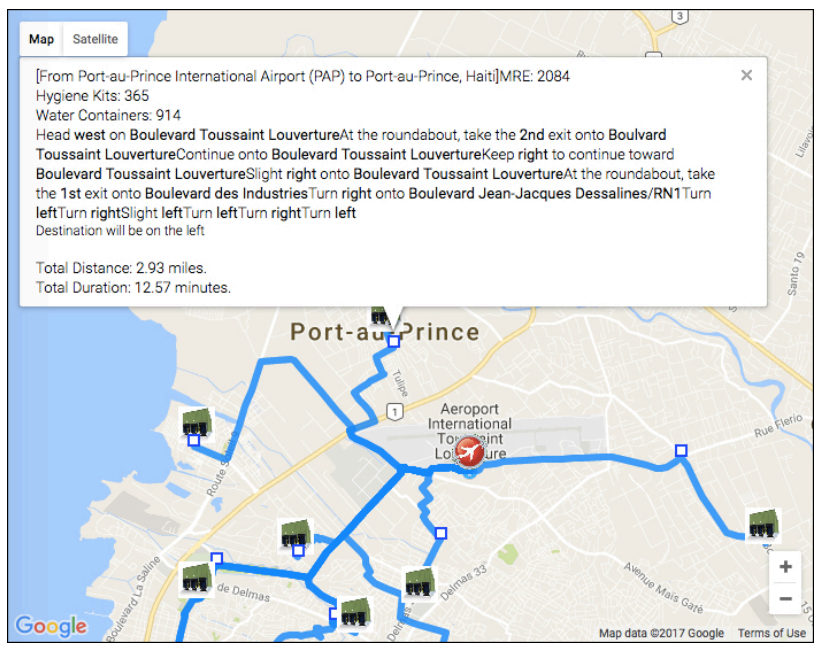

Figure 15: Distribution paths and supply quantities.

for radiological emergencies, rapid screening and decontamination, even among emergency responders and citizens living close to the nuclear plants. During the Fukushima disaster, citizens learned very little through public media or from government officials. The mitigation efforts for radiation exposure and health risks were further clouded as a result of conflicting information released by the Japanese and the US government (e.g., on the radius for evacuation) [26].

First month after March 11, 2011, over 3,000 workers were working around-the-clock on various emergency issues related to the failed nuclear plants. Lack of planning led to missed screening of workers. To facilitate the disaster response effort, RealOpt-Regional was used to determine the sites and the numbers of shelters needed to house the displaced population, while RealOpt-CRC was deployed for rapid screening, decontamination and health registry and monitoring for emergency workers and the affected population.

Figure 16 shows a photo of radiological screening of children displaced from their homes near the Fukushima Daiichi nuclear plants.

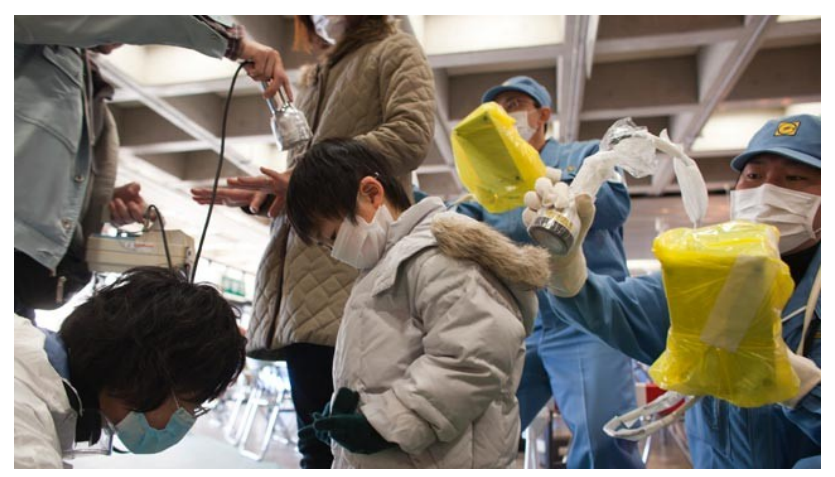

Figure 16: Screening of children for radiation contamination after the 2011 Fukushima Daiichi radiological disasters.

RealOpt-CRC was used to design the optimal process layout for screening and decontamination. Some key tasks include detection of the presence of radioactive contamination on the body 
or clothing, removal of the contamination (decontamination), detection of the intake of radioactive materials into the body, registration/tracking, and medical consultation. The screening sites must include staff members and equipment capable of detecting contamination through beta/gamma portal monitors; monitoring for general contamination using hand-held instruments, fielding questions and addressing all concerns; and distributing event and follow-up information. Further, when internal contamination assessment is to be performed, licensed physicians are needed to carry out the procedure properly. Table 1 contrasts the results using two screening layouts, namely by switching the ordering on the use of portal monitor and hand-held screening instruments. The results underscore the importance of optimizing process design and the resource allocation, which has significant impact on the overall operations efficiency and systems performance.

Table 1: Contrasting throughput for two different layouts for a screening and decontamination center.

\begin{tabular}{lll}
\hline & Layout 1 & Layout 2 \\
\hline Throughput in 12 hours & 52,982 & 40,217 \\
Ave. time spent & 8 minutes & 15 minutes \\
Greeting & 8 workers & 6 workers \\
Paperwork & 8 workers & 6 workers \\
Portal monitor & 16 & 3 \\
Hand-held device & 12 & 43 \\
Decontamination & 46 & 34 \\
Bioassays & 10 stations & 8 stations \\
\hline
\end{tabular}

4.2.3 RealOpt-ASSURE@ $[\mathbf{1}, \mathbf{1 8}, \mathbf{1 9}]$. In Lee et al. [1, 18, 19], we expand the epidemiology module into a general-purpose digital disease surveillance and response system, RealOpt-ASSURE৫. RealOpt-ASSURE allows users to understand the disease dynamics and interplay among hosts and vectors and evaluate the effectiveness of different containment strategies. The model incorporates the life cycles for both hosts and vectors and facilitates the derivation of the basic reproduction number to gain insight on containment strategies. It allows users to contrast the impact and influence of different parameters on the virus trend and outbreak spread. Epidemiologists and public health planners can evaluate different containment strategies and their combination effects to achieve early containment by minimizing total infections. The result can help decision makers select and invest in the strategies that are most effective to combat the infection spread. The RealOpt-ASSURE tool demonstrates the importance of "digital disease surveillance" in response to waves of epidemics including Zika virus, Dengue, Ebola and cholera.

Figure 17 shows a Zika containment analysis that was adopted for implementation. Each square corresponds to a combination strategy. For example, the box labeled "S0" represents the combined strategy of "reducing vector population by $20 \%$, increasing vector mortality by $10 \%$, and reducing biting rate by $5 \%$." We note the many boxes (strategies) that have the same color (total infection). This visualized Pareto frontier offers an economic-decision-framework for policy makers. They can review results, contrast different outcomes, and select a strategy portfolio (with the minimum total infection) that is compatible with their local environment and regional demographics $[1,18]$.

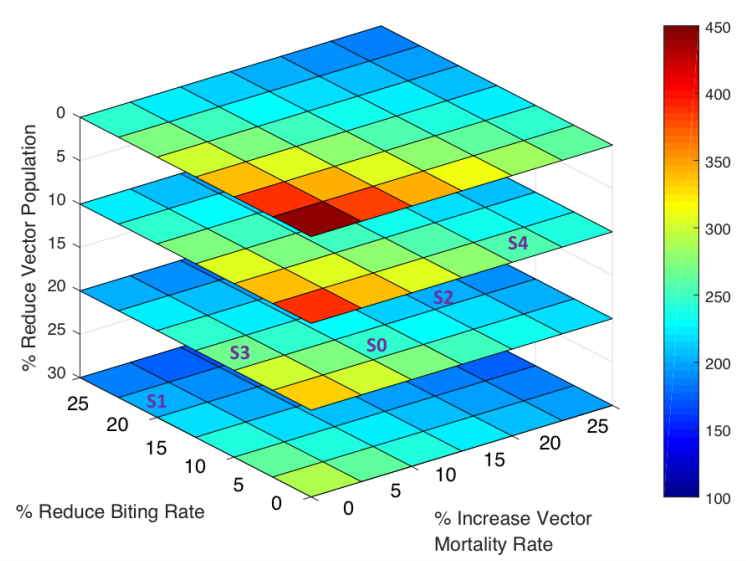

Figure 17: This color-coded figure shows the outcome (total infections) resulted from different containment strategies.

\section{CHALLENGES AND CONCLUSIONS}

Modeling and optimizing public health infrastructure involve elements of resource allocation under risk, uncertainty, and time pressure; large-scale supply-chain management; transportation and operational logistics; and medical treatment and population protection. The operations must be supported by an effective communication infrastructure. There is a necessity for vertical and horizontal integration and communication, where federal, state, local, tribal, territorial, private and business stakeholders work towards a common goal of a resilient public health system. The infrastructure must be flexible, scalable, and sustainable to support an effective and timely response, and to mount rapid recovery and mitigation operations.

In this paper, we discuss a novel interactive web-based public health "digital" informatics decision support system, RealOpt, a suite of tools for real-time emergency preparedness planning, medical countermeasures dispensing, and public health surveillance. While our previous papers on RealOpt focused on the mathematics for POD design and resource allocation, facility location of service network, and disease propagation analysis, this paper focuses on its web-based system design, architecture and functionalities. We also highlight its public health information sharing, reverse reporting, incidents tracking and monitoring, and supply/resupply coordination capabilities. All these elements are critical and essential for successful and effective response operations. We introduce herein RealOpt's usage in the Haiti and Japan Tohoku Disaster response efforts, the Ebola operations for W. Africa, and the Zika containment strategies across various countries in the world. The Tohoku earthquake, tsunami, and its subsequent radiological incidents accentuate the devastating impacts of cascading failures. One specific tragedy underscores the importance of real-time on-the-fly planning and optimization during an unfolding event: On March 11, 30 response officials gathered on the upper floor and roof in the disaster management headquarters for the town of Minamisanriku. 
The tsunami completely flooded the structure and only 11 people survived.

Coupled with Google Maps JavaScript API V3, RealOpt-Regional provides public health emergency preparedness coordinators spatial understanding of the planning region, empowers them in building a mathematical model for optimizing a service network in a graphical visualization environment; and, more importantly, it solves the problem in real time. The client-server architecture not only relieves the need for intensive computation from system users, it also embodies the concept of system modularization and encapsulation to maintain the extendibility of the solution engine without interfering with user experience.

Designing a safe and cost-effective network of service constructs (shelters, medical tents, point-of-dispensing PODs etc.) entails a complex process from planning to actual execution. RealOptRegional requires only a web browser for authorized users to access (including regional commanders, facility planners and managers, and epidemiologists), and provides a common platform for public health workers from different governmental levels (federal/state/local agencies). Using the RealOpt environment, stakeholders crucial to response planning and execution can share information, interact and integrate their respective components for decision/policy making within a trusted shared information environment.

RealOpt has been deployed to public health and emergency planners since December 2009. Currently there are over 200 regional commanders using RealOpt-Regional, most of the users are regional program directors or state response coordinators in Public Health or Emergency Management departments. There are over 10,000 RealOpt-POD site users. RealOpt has been used for response to hurricane, fire, flood, and other public health critical missions including international disaster crises. Its use helps in protecting and saving human lives.

We are currently integrating the standalone RealOpt-Evacuate $\odot$ into the online RealOpt system. RealOpt-Evacuate is a large-scale evacuation system that serves as a strategic-based decision analysis support system in assisting regional evacuation operations, planning and training, in conjunction with population protection (medical countermeasures, medical care, shelters and food supplies) We also distribute a standalone RealOpt-RSS $\odot$ system, a software modeling, simulation and optimization tool for efficient logistics and operations management of Receipt, Stage and Storage (RSS) facilities and regional distribution nodes for medical countermeasures or other emergency supplies. RealOpt-RSS assists in planning quick and efficient strategies for processing medicines and/or equipment from the receiving point of the RSS to RSS vehicles for delivery. The software includes a warehouse design module, and specific computational modules related to the RSS facility, material handling equipment, staffing, and site configuration.

\section{ACKNOWLEDGMENTS}

This work is partially supported by a grant from the Centers for Disease Control and Prevention and the National Science Foundation (IIP-1516074).

\section{REFERENCES}

[1] AAAS. 2017. Adaptable model recommends response strategies for Zika, other pandemics. (2017). https://www.eurekalert.org/pub_releases/2017-02/ giot-amr021717.php

[2] AirNav. 2017. AirNav: Browse Airports. (2017). https://www.airnav.com/ airports/us

[3] K. Alibek and S. Handelman. 1999. Biohazard: The chilling true story of the largest biological weapons program in the world-Told from inside by the man who ran it. John Wiley and Sons, New York.

[4] American School Directory. 2017. American School Directory. (2017). http: //www.asd.com/asd/dshomepage.php

[5] ArcGIS. 2017. ArcGIS. (2017). https://www.arcgis.com

[6] Assisted Senior Living. 2017. Assisted Senior Living. (2017). http://www. assistedseniorliving.net

[7] R. J. Bellamy and A. R. Freedman. 2001. Bioterrorism. QfM 94, 4 (April 2001), 227-234.

[8] Centers for Disease Control and Prevention. 2017. Medical Countermeasure Readiness. (2017). https://www.cdc.gov/phpr/readiness/mcm.html

[9] Google. 2017. Google Maps JavaScript API V3. (2017). https://developers.google. com/maps/documentation/javascript/

[10] Government Accountability Office. 2011. National Capital Region - 2010 strategic plan is generally consistent with characteristics of effective strategies. Technical Report. United States Government Accountability Office, Washington, D.C.

[11] High-Schools. 2017. Data on U.S. Public High Schools and Private High Schools. (2017). https://high-schools.com

[12] Homeless Shelter Directory. 2017. Homeless Shelter Directory. (2017). http: //www.homelessshelterdirectory.org

[13] Hotel Rooms. 2017. Hotel Rooms. (2017). http://www.hotelrooms.com

[14] E. H. Kaplan, D. L. Craft, and L. M. Wein. 2002. Emergency response to a smallpox attack: the case for mass vaccination. Proc Natl Acad Sci 99, 16 (August 2002), 10935-10940.

[15] J. Lawler and C. Mecher. 2007. White house homeland security council, private communication. (2007)

[16] E. K. Lee, C.-H. Chen, F. H. Pietz, and B. Benecke. 2009. Modeling and optimizing the public-health infrastructure for emergency response. Interfaces 39, 5 (October 2009), 476-490.

[17] E. K. Lee, C.-H. Chen, F. H. Pietz, and B. Benecke. 2010. Disease propagation analysis and mitigation strategies for effective mass dispensing. AMIA Annu Symp Proc 2010 (November 2010), 427-432.

[18] E. K. Lee, Y. Liu, and F. H. Pietz. 2016. A compartmental model for Zika virus with dynamic human and vector populations. AMIA Annu Symp Proc 2016 (November 2016), 743-752.

[19] E. K. Lee, Y. Liu, and F. H. Pietz. 2017. A computational framework for a digital surveillance and response tool: application to avian influenza. Technical Report. Georgia Institute of Technology, Atlanta, Georgia.

[20] E. K. Lee, S. Maheshwary, J. Mason, and W. Glisson. 2006. Decision support system for mass dispensing of medications for infectious disease outbreaks and bioterrorist attacks. Ann Oper Res 148, 1 (November 2006), 25-53.

[21] E. K. Lee, S. Maheshwary, J. Mason, and W. Glisson. 2006. Large-scale dispensing for emergency response to bioterrorism and infectious-disease outbreak. Interfaces 36, 6 (December 2006), 591-607.

[22] E. K. Lee, F. H. Pietz, B. Benecke, J. Mason, and G. Burel. 2013. Advancing public health and medical preparedness with operations research. Interfaces 43 , 1 (February 2013), 79-98.

[23] E. K. Lee, H. K. Smalley, Y. Zhang, F. H. Pietz, and B. Benecke. 2009. Facility location and multi-modality mass dispensing strategies and emergency response for biodefence and infectious disease outbreaks. Int 7 Risk Assess Manag 12, 2-4 (2009), 311-351.

[24] E. K. Lee and M. Zaider. 2008. Operations research advances cancer therapeutics. Interfaces 38, 1 (January 2008), 5-25.

[25] J. Mason and M. Washington. 2003. Optimizing staff allocation in large-scale dispensing centers. Technical Report. Centers for Disease Control and Prevention, Atlanta, Georgia.

[26] D. Normile. 2011. Conflicting U.S. and Japan evacuation policies sow confusion. (March 2011). http://www.sciencemag.org/news/2011/03/ conflicting-us-and-japan-evacuation-policies-sow-confusion

[27] S. Scott and C. J. Duncan. 2001. Biology of plagues: evidence from historical populations. Cambridge University Press, Cambridge.

[28] U.S. Census Bureau. 2017. Data Tools and Apps. (2017). https://www.census. gov/data/data-tools.html

[29] U.S. Department of Education. 2017. The Database of Accredited Postsecondary Institutions and Programs. (2017). https://ope.ed.gov/accreditation

[30] L. M. Wein, D. L. Craft, and E. H. Kaplan. 2003. Emergency response to an anthrax attack. Proc Natl Acad Sci 100, 7 (April 2003), 4346-4351.

[31] Zipmap. 2017. Zip Code Boundary Maps for all US States and Possessions. (2017). http://www.zipmap.net 